\title{
A Very Low Bit Rate Video Coding System Using Adaptive Region-Classified Vector Quantization
}

\author{
Yee-Wen Chen, Liang-Gee Chen and Mei-Juan Chen \\ Department of Electrical Engineering \\ National Taiwan University, Taipei, Taiwan, R.O.C.
}

\begin{abstract}
In this paper, a new video coding algorithm suitable for the very low bit rate video application is presented. It takes advantage of the prior knowledge of the image type to segment the image into different regions, then codes each region with different coding criterion and method according to the different importance. An adaptive region-classified vector quantization strategy is also exploited in this algorithm. With the segmentation of the frame and high correlation between frames, better codebooks of vector quantization are constructed to improve the quality. According to the simulation results, acceptable quality at about $10 \mathrm{kbits}$ per second can be obtained for the typical test sequences.
\end{abstract}

\section{Introduction}

Recently a new interest has been arisen in the field of the very low bit rate video application. The motivation of this new interest lies in the development of new applications such as videophones, video conferencing, and many others. The major requirements for these applications are the low capacity for transmission and storage, in order to use the existing Public Switched Telephone Networks (PSTN) or mobile channels.

Numerous algorithms have been explored to implement the high compression system, such as modelbased [1] and object-based [2] methods. However, above two methods were involved with complicated computation and irreqular data flow, which made real-time implementation difficult.

\section{Segmentation-Based Algorithm}

In this paper, we consider subjective view, compression ratio, and hardware complexity to propose a segmentation-based algorithm suitable for the high compression video application. Figure 1 shows the overall video coding system. It mainly consists of three parts : The segmentation, codebook generation and coding parts. With the prior knowledge of the image type, the segmentation part segments the current frame into different regions, especially the

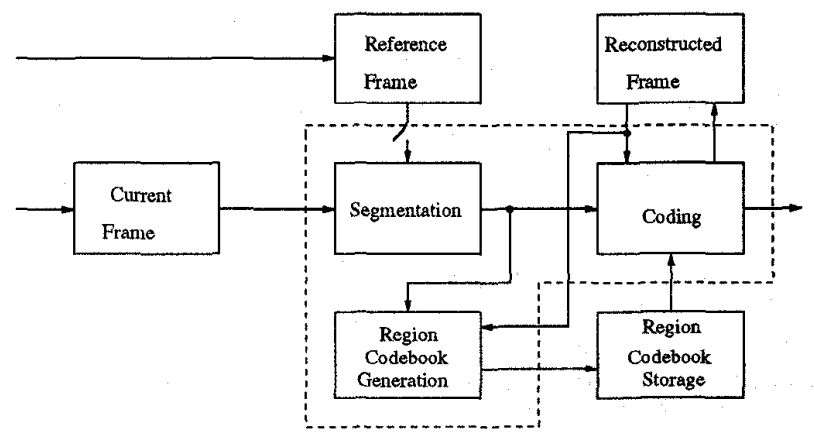

Figure 1: The Block Diagram of the Video Coding System

face region from the other regions. Since observer$s$ tend to concentrate on the face region, a careful coding method should be adopted for a better subjective view. In addition, it is believed that better results can be obtained for vector quantization in the inside training manner. Because of high correlation for videophone or video conference sequences, using decoded images as training image basis is similar to the inside training condition. By combining with the concept of region, an adaptive region-classified vector quantization strategy is used as the main profile of the video coding system. The codebook generation part generates the region codebooks according to the region information from the segmentation part and each decoded frame. The coding part performs the coding-decoding process with those region codebooks generated from codebook generation part.

There is a setup period at the beginning of the transmission of the images, which lasts approximately several seconds. During this period, the encoder sends an intraframe to the decoder, and uses this frame to construct all of the initial region codebooks. At the same time, the encoder segments this frame and sends the side informations of regions to the decoder to provide the informations for region codebook generation. Some parts of the succeeding frames can be reconstructed using those region codebooks. 


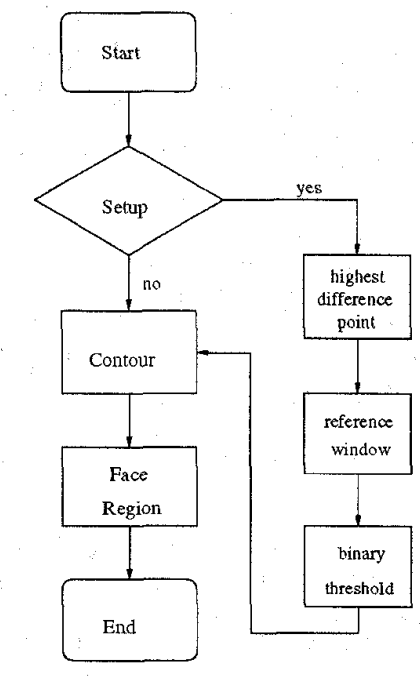

Figure 2: The Flow Chart of the Segmentation Algorithm

\subsection{Segmentation}

Figure 2 shows the flow chart of the segmentation algorithm. It finds the contours in the reference window which is constructed either by the previous frame or another heuristic method for normal or setup period respectively. During the setup period, since the face region is unknown, extra process of 'highest difference point' is executed to find the top of the head. The reference window which provides an approximate location of face region is constructed according to this top point. The binary threshold is computed only during the setup period because of the high correlation between frames, thus bit map of each frame can be obtained to provide the contour tracing basis. At last, the face region and the further segmentation are obtained by detecting the boundaries of the most suitable contour.

\subsection{Codebook Generation}

The most popular method for the codebook generation of vector quantization is LBG [3] algorithm, which is good but time-consuming. Here a codebook generation method for on-line training is presented, as shown in Figure 3. For each region, given the initial codebook, the following actions are executed for each training word.

1. Given the codebook, $C_{n}$, update the distance table which records the distance relations between any two codewords, and find the minimum distance from the values in the distance table.

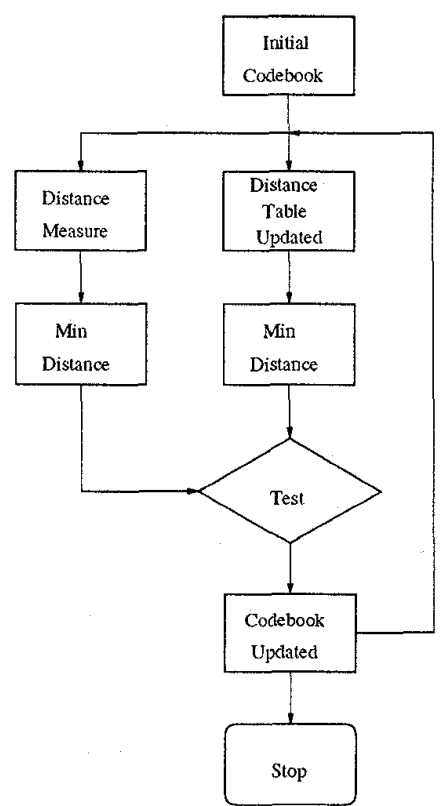

Figure 3: The Flow Chart of the Codebook Generation

2. Given the $n+1$ 'th training word, compute the distances to the codewords, and find the minimum distance of these distances.

3. Preform the test between values obtained in step 1 and step 2. If the value obtained in step 1 is larger, merge $n+1$ 'th training word with the codeword closest to it as a new codeword. Otherwise, merge those two closest codewords as one new codeword and use the $n+1$ 'th training word as another new one.

The block size of $4 \times 4$ and $8 \times 8$ is applied for the face and the other regions respectively. The training set of each region for the setup period is generated by shifting the partition position by $0,1,2,3$ and 0,2 , 4,6 for the block size of $4 \times 4$ and $8 \times 8$ respectively in the horizontal and vertical directions, thus results in 16 times the size of original region as the training image. For the normal condition, if the block with its error part is coded, it is added to the training set. The codebook will be adapted when a frame has been coded.

\subsection{Coding}

Figure 4 shows the flow chart of the coding algorith$\mathrm{m}$. The coding process classifies each block to motion ( Motion-Compensation [4]), still, or intra (MotionFailure) block. For each MF-block, the adaptive region-classified vector quantization is applied. For the face region, a detail-classified method is used to 


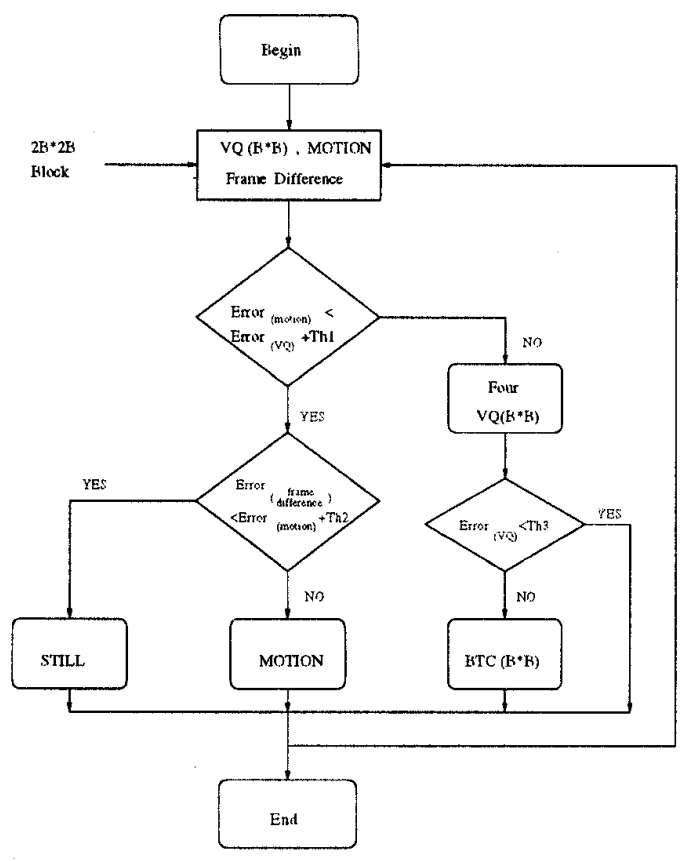

Figure 4: The Flow Chart of the Coding Algorithm

further segment the eye part and mouth part from the face part. If the error distortion of the MF-block is larger than a perset threshold, the block truncation coding [5] is applied to improve the performance. The information for motion compensation and block truncation coding is further compressed by the method of variable-length coding ( VLC).

\section{Simulation Results}

In this experiment, three sequences, 'Miss America', 'Elsa' and 'C.M.J.' are tested, and the frame number for each sequence is 47,51 , and 530 respectively. As the segmentation algorithm described above, the segmentation result of 'Miss America' is shown in Figure 5. Figure (a) (e) shows the intersection between the reference window and the original frame for setup time or normal time respectively. Figure (b) (f) shows the contours with some parts inside the reference window, and the parts of the contour exceeding the reference window are traced also. Figure (c) (g) shows the face region of the frame. Figure (d) (h) shows the further segmentation of the frame, that is, the frame is further segmented into eye region, mouth region, left shoulder region, right shoulder region, breast region and background.

For each region, the codebook size is approximately a specific percentage of the size of training words. There are more intra blocks in the face region because the eye part and mouth part of the face region
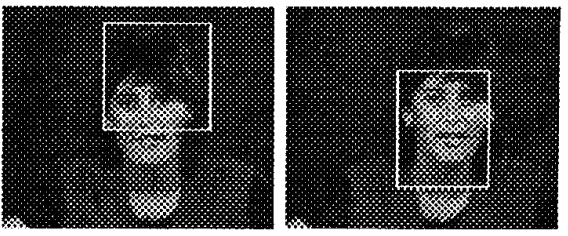

(a)(e) Reference window
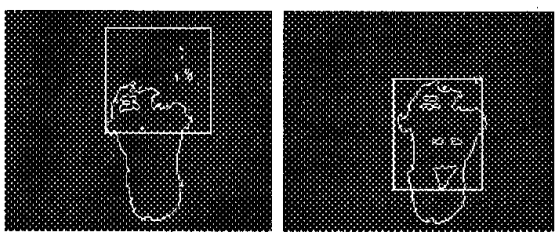

(b)(f) Contours

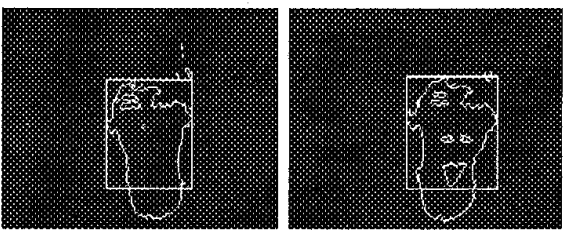

(c)(g) Face region
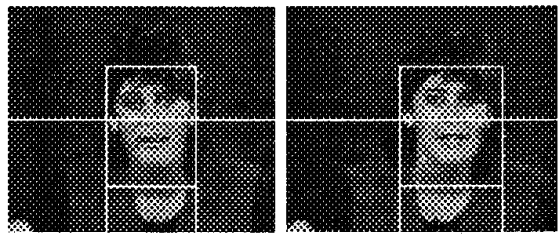

(d)(h) Segmentation result

Figure 5: Segmentation Result for Miss America (Setup Period or Normal Condition)

correspond to the motion failure part of the image, and also the coding criterions for these regions are more strict in order to keep a good subjective view in the face region. The average bits and average $P$ SNR for a frame is recorded for the face region and overall region respectively, as listed in Table 1 . Figure 6 shows the result of PSNR and bits vesus frame number for the face region and overall region respectively for 'Miss America' sequence. A comparison is made between the proposed algorithm and the CCITT H.261 [6], as shown in Table 2, and Figure 7 for the 'Miss America' sequence. According to the experimental results, acceptable quality around $10 \mathrm{k}$ bits per second can be obtained for the typical test sequences.

\section{Conclusion}

In this paper, a new algorithm for the very low bit rate video coding system is presented. This algorith$m$ contains segmentation, codebook generation, and coding parts. The segmentation part segments the 


\begin{tabular}{|l|c|c|c|c|}
\hline & \multicolumn{2}{|c|}{ Overall Region } & \multicolumn{2}{c|}{ Face Region } \\
\hline & PSNR(dB) & Bits(bps) & PSNR(dB) & Bits(bps) \\
\hline Miss & 35.16 & $10.7 \mathrm{k}$ & 31.18 & $8.4 \mathrm{k}$ \\
\hline Elsa & 33.87 & $10.8 \mathrm{k}$ & 31.18 & $7.9 \mathrm{k}$ \\
\hline C.M.J. & 33.87 & $9.9 \mathrm{k}$ & 33.09 & $8.2 \mathrm{k}$ \\
\hline
\end{tabular}

Table 1: Performance of the test sequences

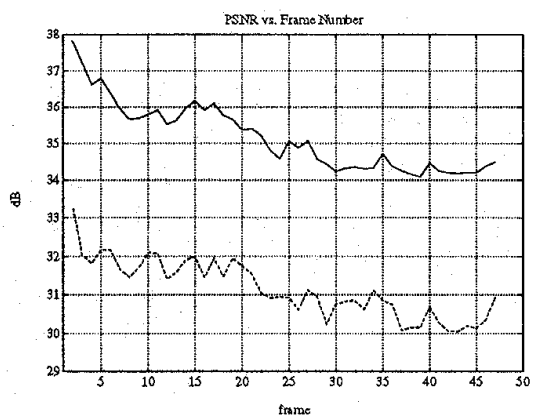

(a) PSNR vs frame number

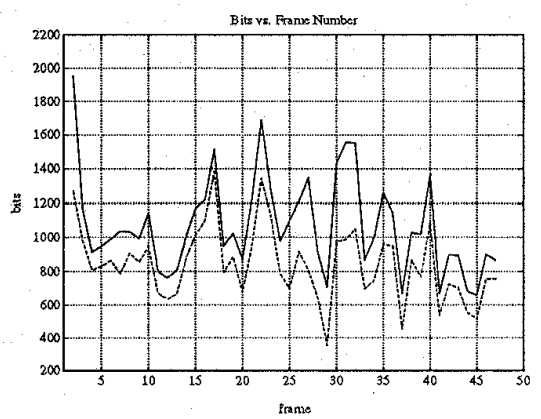

(b) Bits vs frame number

Figure 6: Coding Result of "Miss America"

\begin{tabular}{|l|c|c|}
\hline & H.261 & Proposed \\
\hline coding of the residtal erroc & DCT+Q & none \\
\hline coding of the region with large residual & DCT+Q & vQ+BTC \\
\hline priocity policy & no & yes \\
\hline prioc knowlidge & no & yes \\
\hline sdaptive strategy & no & yes \\
\hline tempocal corcelation utilization for inta btock & no & yes \\
\hline
\end{tabular}

Table 2: Comparisons with the H.261

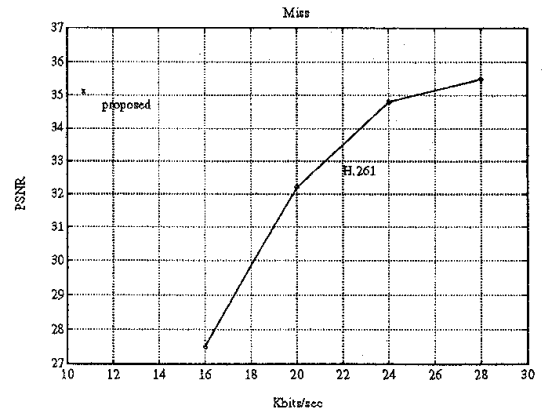

Figure 7: Comparisons Between Proposed Algorithm and H.261 for "Miss America"

current frame to different regions of different importance. The codebook generation part generates the region codebooks from each decoded frame and segmentation informations in an adaptive manner. The coding part performs more detail coding in the more important region according to the region informations and region codebooks. The experimental results show that the image quality is acceptable at about the transmission rate of $10 \mathrm{k}$ bits per second.

\section{Reference}

[1] P. Gerken," Object-Based Analysis-Synthesis Coding of Image Sequences at Very Low Bit Rates," IEEE Trans. on Circuits and Systems for Video Tech. pp. 228-235, June 1994.

[2] C. S. Choi and K.Aizawa," Analysis and Synthesis of Facial Image Sequences in Model-Based Image Coding," IEEE Trans. on Circuits and Systems for Video Tech. pp. 257-275 ,June 1994.

[3] Y. Linde, A. Buzo and R.M. Gray, " An algorith$m$ for vector quantizer design,"IEEE Trans. Commun,Vol. COM-28, pp. 84-95, January 1980.

[4] J. R. Jain and A.K. Jain," Displacement Measurement and its Application in Interframe Image Coding," IEEE Trans. Commun, vol. COM-29, pp. 1799-1808, Dec. 1981.

[5] Delp. E.J. and Mitchell, O.R., " Image Conpression Using Block Truncation Coding," IEEE Trans. Communications. vol. COM-27, Sept. 1979, pp. 1335-1342.

[6] International Telegraph and Telephone Consultative Committee (CCITT), Video codec for visual services at $\mathrm{p} 64 \mathrm{~kb} / \mathrm{s}$, CCITT H.261 Recommendation, CDM XV-R 37-E, Aug. 1990. 